\title{
Impact of the Agents Treated for Metabolic Syndrome on Surgical Complications
}

\author{
Sheng-Lung Hsu'1,2*, Chiu-Lan Chen², Kuo-Sheng Liü ${ }^{2}$ and Jin-Chang \\ $\mathrm{Hsieh}^{3}$ \\ ${ }^{1}$ Department of Surgery, Chi Mei Hospital Chiali, Taiwan \\ ${ }^{2}$ Department of Pharmacy, Chia Nan University of Pharmacy \& Science, Taiwan \\ ${ }^{3}$ Research Center for Testing and Assessment, National Academy for Educational \\ Research, Taiwan
}

*Corresponding author: Sheng-Lung Hsu, Minister of the Department of Surgery, Chi Mei Hospital Chiali, No.606, Jialixing, Jiali District, Tainan City 722, Taiwan, Tel: 886-929767335; Email: 900249@mail.chimei.org.tw

\section{Abstract}

Background: The impact of metabolic syndrome (MS) on different surgical operations has gained significant attention recently. However, to date, it is unclear whether the medications used to treat MS can have an effect on surgical complications.

Objectives: In this study, we aimed to investigate the effects of therapeutic agents used for the treatment of metabolic syndrome on surgical complications.

Methods: We performed a retrospective case-control study based on chart reviews of 2624 surgical patients at our hospital in 2016. Data regarding surgical complications obtained from the hospital records were analyzed using binary logistic regression. The independent variables were angiotensin-converting-enzyme inhibitor, angiotensin receptor blocker (ARB), dihydropyridine calcium-channel blockers (DCCB), diuretics, potassium-sparing diuretics, nondihydropyridine calcium-channel blockers (NDCCB), dipeptidyl peptidase-4 inhibitor, glucagon-like peptide-1 receptor agonists (GLP1 agonists), nonselective beta-blockers, selective beta-blockers, peripheral vasodilators, alpha-glucosidase inhibitors, insulin, metformin, sulfonylureas, thiazolidinediones, bile acid sequestrants, fibrates, and statins. The dependent variable was surgical complications. Among the surgical patients included in the study, 328 (12.5\%) had a history of metabolic syndrome.

Results: In diseases with the same severity score [ $\geq$ ASA 3(American Society of Anesthesiologists)], diuretics, NDCCB, DCCB, insulin, GLP1 agonists, and selective beta-blockers had a significant influence in univariate logistic regression. However, multivariate logistic regression using control variables showed that only diuretic use was statistically significant (odds ratio 1.968, 95\% confidence interval 1.141-3.394, $\mathrm{p}=0.015$ ). 
Conclusion: Several medications used for the treatment of metabolic syndrome pose a risk for surgical complications, although only diuretics showed a significant association.

Keywords: Medication; Metabolic Syndrome; Odds Ratio; Risk Factor; Surgical Complication

Abbreviations: MS: Metabolic Syndrome, ARB: Angiotensin Receptor Blocker, ASA: American Society of Anesthesiologists, ICD: International Classification of Diseases, WC: Waist Circumference, BMI: Body Mass Index, WHO: World Health Organization, ACEi: Angiotensin-Converting-Enzyme inhibitor, DCCB: Dihydropyridine Calcium-Channel Blockers, NDCCB: NonDihydropyridine Calcium-Channel Blockers, DPP4i: Dipeptidyl Peptidase-4 inhibitor, GLP1 Agonists: Glucagon-Like Peptide-1 Receptor Agonists, OR: Odds Ratio, CI: Confidence Interval, SSIs: Surgical Site Infections.

\section{Introduction}

Although surgical complications are common in the daily practice of surgeons, they are considered serious issues related to surgery [1], which exacerbate the suffering of patients and lead to lengthy hospital stays and higher medical expenses. In recent years, the impact of metabolic syndrome on different surgical operations has gained significant attention [2-7]. Several risk factors that could affect convalescence, including age, gender, disease history, physical status of the patient, anesthesia, blood loss, and wound condition, have been reported.

The prevalence of metabolic syndrome has been steadily increasing in Taiwan in recent years [8]. Metabolic syndrome can result in vascular thromboembolism of the tissue [9], which could lead to interruption of blood supply to wounds. However, there has been little research on the relationship between medications prescribed for metabolic syndrome and surgical complications except one case-control study about ARB. We aimed to investigate the effects of therapeutic agents used for the treatment of metabolic syndrome on surgical complications and to identify the agents that have a significant effect on surgical complications.

\section{Material \& Methods}

This study is a correlational, case-control study using data from retrospective chart review. The main outcome of all subjects who received drugs for the treatment of metabolic syndrome was compared to patients without metabolic syndrome. The study was approved and monitored by the International Review Board of the XXXXX medical center and the requirement for informed consent waived. In total, 2624 surgical patients in our regional hospital between January 2016 and December 2016 with a mean age of 57.7 years $(S D=18.85)$ were included in our study after removing repetitive surgical data. The surgical complications were identified by the hospital surveillance system as follows: [International Classification of Diseases (ICD)-9 codes 457.0, 519.00519.09 , 536.40-536.49, 564.2, 569.60-569.69, 674.12, $674.14,674.32,674.34,996.00-996.09,996.1,996.2$, 996.4, 996.51-996.59, 996.60-996.63, 996.66-996.69, 996.71-996.79, 996.80-996.89, 996.90-996.99, 997.00997.99, 998.0,998.1, 998.2, 998.3, 998.4, 998.51, 998.59, 998.6, 998.7, 998.81-998.9, 998.9]. All the above codes correlated with surgical complication in different body systems. As waist circumference (WC) is not measured routinely in our clinical practice, we chose body mass index (BMI) as a parameter indicating overall obesity. We modified the definition of metabolic syndrome after referring to the criteria of the Health Promotion Administration, Ministry of Health and Welfare (Table 1) [10] and the World Health Organization (WHO) $[11,12]$.

\begin{tabular}{|l|}
\hline Any three of the following: \\
\hline Use of anti-dyslipidemic medication \\
\hline $\begin{array}{l}\text { Use of anti-hypertensive medication or history of } \\
\text { hypertension }\end{array}$ \\
\hline $\begin{array}{l}\text { Use of anti-diabetic medication or history of diabetic } \\
\text { mellitus }\end{array}$ \\
\hline BMI $>30$ \\
\hline $\begin{array}{l}\text { Men with HDL }<40 \mathrm{mg} / \mathrm{dL} \text {, women with } \mathrm{HDL}<50 \\
\mathrm{mg} / \mathrm{dL}\end{array}$ \\
\hline
\end{tabular}

Table 1: Criteria for diagnosis of metabolic syndrome.

\section{Statistical Methods}

All statistical analyses were performed with SPSS 24.0 (IBM Corporation, Armonk, NY, USA). The data were analyzed by binary logistic regression. The independent variables were angiotensin-converting-enzyme inhibitor (ACEi), ARB, dihydropyridine calcium-channel blockers (DCCB), diuretics, potassium-sparing (K+-sparing) diuretics, non-dihydropyridine calcium-channel blockers (NDCCB), dipeptidyl peptidase-4 inhibitor (DPP4i,), glucagon-like peptide- 1 receptor agonists (GLP1 agonists), nonselective beta-blockers, selective beta-blockers, 
peripheral vasodilators, alpha-glucosidase inhibitors, insulin, metformin, sulfonylureas, thiazolidinediones, bile acid sequestrants, fibrates, and statins. The dependent variable was surgical complication. We also selected several significant variables including hypertension, diabetes mellitus, dyslipidemia, age, gender, BMI, and ASA (American Society of Anesthesiologists) as control variables to perform multivariate logistic regression.

\section{Results}

Three hundred and twenty-eight (12.5\%) patients had a history of metabolic syndrome. All subjects with metabolic syndrome (odds ratio (OR) $=2.698$, 95\% confidence interval (CI) 1.695-4.296; p < 0.001) or any disease of metabolic syndrome had larger odds for surgical complications $(\mathrm{p}<0.05)$. The elderly surgical patients had more complications than the younger patients. According to the ASA physical status classification, patients with severe disease ( $\geq$ ASA3) had more surgical complications $(\mathrm{p}<0.001)$. Table 2 shows the relationship between agents used for the treatment of metabolic syndrome and surgical complications in all patients. In patients with metabolic syndrome, the agents showing a correlation with surgical complications were diuretics, NDCCB, insulin, and GLP1 agonists (Table 3). In patients with severe diseases ( $\geq$ ASA3), the agents showing a correlation with surgical complications were diuretics, NDCCB, DCCB, selective beta-blockers, insulin, and GLP1 agonists (Table 4). In multivariate logistic regression using control variables, only diuretics showed statistical significance $(\mathrm{OR}=1.968,95 \%$ CI 1.141-3.394; $\mathrm{p}$ $=0.015)$ (Table 5).

\begin{tabular}{|c|c|c|c|c|}
\hline \multirow{2}{*}{ Drugs } & $\begin{array}{c}\text { Patients with surgical } \\
\text { complications (n =97) }\end{array}$ & $\begin{array}{c}\text { Patients without surgical } \\
\text { complications (n =2527) }\end{array}$ & Odds ratio (95\% CI) & p value \\
\cline { 2 - 4 } & $\mathbf{( n \% )}$ & $\mathbf{( n \% )}$ & & \\
\hline ACEi & $5(5.2)$ & $57(2.3)$ & $2.355(0.922-6.014)$ & 0.073 \\
\hline ARB & $24(24.7)$ & $441(17.5)$ & $3.927(2.599-5.933)$ & $<0.001$ \\
\hline DCCB & $44(45.4)$ & $463(18.3)$ & $3.128(2.062-4.475)$ & $<0.001$ \\
\hline Diuretics & $40(41.2)$ & $84(3.3)$ & $2.262(1.017-5.030)$ & 0.045 \\
\hline K$^{+}$-sparing diuretics & $7(7.2)$ & $118(4.7)$ & $3.734(2.090-6.674)$ & $<0.001$ \\
\hline NDCCB & $15(15.5)$ & $91(3.6)$ & $2.406(1.133-5.110)$ & 0.022 \\
\hline Nonselective beta-blockers & $8(8.2)$ & $118(4.7)$ & $1.588(0.720-3.502)$ & 0.252 \\
\hline Peripheral vasodilators & $7(7.2)$ & $328(13.0)$ & $3.618(2.347-5.578)$ & $<0.001$ \\
\hline Selective beta-blockers & $34(35.1)$ & $61(2.4)$ & $2.197(0.862-5.597)$ & 0.099 \\
\hline Alpha-glucosidase inhibitors & $5(5.2)$ & $182(7.2)$ & $2.738(1.588-4.721)$ & $<0.001$ \\
\hline DPP4i & $17(17.5)$ & $285(11.3)$ & $4.643(3.020-7.137)$ & $<0.001$ \\
\hline Insulin & $36(37.1)$ & $70(2.8)$ & $8.550(4.909-14.892)$ & $<0.001$ \\
\hline GLP1 agonists & $19(19.6)$ & $206(8.2)$ & $2.226(1.278-3.876)$ & 0.005 \\
\hline Metformin & $16(16.5)$ & $129(5.1)$ & $1.446(0.657-3.183)$ & 0.36 \\
\hline Sulfonylureas & $7(7.2)$ & $26(1.0)$ & $3.070(0.913-10.323)$ & 0.07 \\
\hline Thiazolidinediones & $3(3.1)$ & $24(0.9)$ & $2.196(0.511-9.426)$ & 0.29 \\
\hline Bile acid sequestrants & $2(2.1)$ & $19(0.8)$ & $7.174(2.621-19.635)$ & $<0.001$ \\
\hline Fibrates & $5(5.2)$ & $169(6.7)$ & $1.604(0.818-3.143]$ & 0.169 \\
\hline Statin (high intensity) & $10(10.3)$ & $31(1.2)$ & $0.839(0.113-6.208)$ & 0.863 \\
\hline Statin (low intensity) & $1(1.0)$ & $61(2.4)$ & $1.739(0.619-4.883)$ & 0.294 \\
\hline Statin (moderate intensity) & $4(4.1)$ & & & \\
\hline
\end{tabular}

Table 2: Univariate logistic regression analysis showing the relationship between medications used to treat metabolic syndrome and surgical complications in all patients. 


\begin{tabular}{|c|c|c|c|c|}
\hline \multirow{2}{*}{ Drugs } & $\begin{array}{c}\text { Patients with surgical } \\
\text { complications (n =26) }\end{array}$ & $\begin{array}{c}\text { Patients without surgical } \\
\text { complications (n =302) }\end{array}$ & Odds ratio (95\% CI) & \multirow{2}{*}{ p value } \\
\cline { 2 - 4 } & $\mathbf{\text { (n\%) }}$ & $\mathbf{\text { (n) }}$ & & \\
\hline ACEi & $3(11.5)$ & $23(7.6)$ & $1.582(0.442-5.668)$ & 0.481 \\
\hline ARB & $12(46.2)$ & $167(55.3)$ & $0.693(0.310-1.548)$ & 0.371 \\
\hline DCCB & $11(42.3)$ & $122(40.4)$ & $1.082(0.481-2.435)$ & 0.849 \\
\hline Diuretics & $16(61.5)$ & $118(39.1)$ & $2.495(1.095-5.683)$ & 0.03 \\
\hline $\mathrm{K}^{+}$-sparing diuretics & $3(11.5)$ & $25(8.3)$ & $1.445(0.406-5.151)$ & 0.57 \\
\hline NDCCB & $7(26.9)$ & $35(11.6)$ & $2.811(1.103-7.162)$ & 0.03 \\
\hline Nonselective beta-blockers & $2(7.7)$ & $18(6.0)$ & $1.315(0.288-6.006)$ & 0.724 \\
\hline Peripheral vasodilators & $4(15.4)$ & $42(13.9)$ & $1.126(0.369-3.429)$ & 0.835 \\
\hline Selective beta-blockers & $7(26.9)$ & $96(31.8)$ & $0.791(0.321-1.944)$ & 0.609 \\
\hline Alpha glucosidase inhibitors & $4(15.4)$ & $46(15.2)$ & $1.012(0.333-3.072)$ & 0.983 \\
\hline DPP4i & $11(42.3)$ & $137(45.4)$ & $0.883(0.393-1.986)$ & 0.764 \\
\hline Insulin & $20(76.9)$ & $145(48.0)$ & $3.609(1.410-9.238)$ & 0.007 \\
\hline GLP1 agonists & $9(34.6)$ & $38(12.6)$ & $3.678(1.531-8.837)$ & 0.004 \\
\hline Metformin & $11(42.3)$ & $138(45.7)$ & $0.871(0.388-1.960)$ & 0.739 \\
\hline Sulfonylureas & $4(15.4)$ & $90(29.8)$ & $0.428(0.143-1.278)$ & 0.129 \\
\hline Thiazolidinediones & $1(3.8)$ & $21(7.0)$ & $0.535(0.069-4.147)$ & 0.55 \\
\hline Bile acid sequestrants & $1(3.8)$ & $13(4.3)$ & $0.889(0.112-7.079)$ & 0.912 \\
\hline Fibrates & $3(11.5)$ & $15(5.0)$ & $2.496(0.673-9.252)$ & 0.171 \\
\hline Statin (high intensity) & $7(26.9)$ & $118(39.1)$ & $0.574(0.234-1.409)$ & 0.226 \\
\hline Statin (low intensity) & $1(3.8)$ & $15(5.0)$ & $0.765(0.097-6.035)$ & 0.8 \\
\hline Statin (moderate intensity) & $3(11.5)$ & $41(13.6)$ & $0.830(0.239-2.891)$ & 0.77 \\
\hline
\end{tabular}

Table 3: Univariate logistic regression analysis showing the relationship between medications used to treat metabolic syndrome and surgical complications in patients diagnosed with metabolic syndrome.

\begin{tabular}{|c|c|c|c|c|}
\hline Drugs & $\begin{array}{c}\begin{array}{c}\text { Patients with surgical } \\
\text { complications } \mathrm{n}=63\end{array} \\
\text { (n\%) }\end{array}$ & $\begin{array}{l}\text { Patients without surgical } \\
\text { complications } \mathrm{n}=1252\end{array}$ & Odds ratio $(95 \% \mathrm{CI})$ & $\begin{array}{c}\mathbf{p} \\
\text { value }\end{array}$ \\
\hline ACEi & $3(4.8)$ & $50(4.0)$ & $1.202(0.364-3.965)$ & 0.763 \\
\hline $\mathrm{ARB}$ & $17(27.0)$ & $293(23.4)$ & $1.210(0.683-2.142)$ & 0.514 \\
\hline DCCB & $28(44.4)$ & $318(25.4)$ & $2.350(1.407-3.925)$ & 0.001 \\
\hline Diuretics & $30(47.6)$ & $321(25.6)$ & $2.637(1.583-4.393)$ & $<0.001$ \\
\hline $\mathrm{K}^{+}$-sparing diuretics & $6(9.5)$ & $62(5.0)$ & $2.020(0.839-4.867)$ & 0.117 \\
\hline NDCCB & $10(15.9)$ & $96(7.7)$ & $2.272(1.120-4.608)$ & 0.023 \\
\hline Nonselective beta blockers & $6(9.5)$ & $59(4.7)$ & $2.128(0.882-5.136)$ & 0.093 \\
\hline Peripheral vasodilators & $3(4.8)$ & $85(6.8)$ & $0.686(0.211-2.235)$ & 0.532 \\
\hline Selective beta-blockers & $20(31.7)$ & $223(17.8)$ & $2.146(1.238-3.719)$ & 0.006 \\
\hline Alpha glucosidase inhibitors & $4(6.3)$ & $51(4.1)$ & $1.579(0.558-4.566)$ & 0.383 \\
\hline DPP4i & $7(11.1)$ & $132(10.5)$ & $1.061(0.474-2.375)$ & 0.886 \\
\hline Insulin & $22(34.9)$ & $215(17.2)$ & $2.588(1.511-4.434)$ & 0.001 \\
\hline GLP1 agonists & $6(9.5)$ & $40(3.2)$ & $3.189(1.299-7.831)$ & 0.011 \\
\hline Metformin & $11(17.5)$ & $157(12.5)$ & $1.475(0.745-2.888)$ & 0.256 \\
\hline Sulfonylureas & $5(7.9)$ & $102(8.1)$ & $0.972(0.381-2.477)$ & 0.952 \\
\hline Thiazolidinediones & $1(1.6)$ & $22(1.8)$ & $0.902(0.120-6.799)$ & 0.92 \\
\hline Bile acid sequestrants & $2(3.2)$ & $16(1.3)$ & $2.533(0.570-11.264)$ & 0.222 \\
\hline Fibrates & $2(3.2)$ & $14(1.1)$ & $2.899(0.645-13.042)$ & 0.165 \\
\hline Statin (high intensity) & $7(11.1)$ & $115(9.2)$ & $1.236(0.550-2.775)$ & 0.608 \\
\hline Statin (low intensity) & $1(1.6)$ & $21(1.7)$ & $0.945(0.125-7.143)$ & 0.957 \\
\hline Statin (moderate intensity) & $3(4.8)$ & $44(3.5)$ & $1.373(0.414-4.548)$ & 0.604 \\
\hline
\end{tabular}

Table 4: Univariate logistic regression analysis showing the relationship between medications used to treat metabolic syndrome and surgical complications in patients with ASA score $\geq 3$. 


\section{Open Access Journal of Urology \& Nephrology}

\begin{tabular}{|c|c|c|c|}
\hline Drugs & Odds ratio & $\mathbf{9 5 \%}$ CI & p value \\
\hline NDCCB & 1.429 & $(0.660-3.097)$ & 0.365 \\
\hline DCCB & 1.697 & $(0.988-2.916)$ & 0.055 \\
\hline ARB & 0.945 & $(0.503-1.775)$ & 0.861 \\
\hline ACEi & 0.919 & $(0.269-3.141)$ & 0.892 \\
\hline Diuretics & 1.968 & $(1.141-3.394)$ & 0.015 \\
\hline K$^{+}$-sparing diuretics & 1.461 & $(0.587-3.636)$ & 0.415 \\
\hline Nonselectivebeta-blockers & 1.322 & $(0.457-3.824)$ & 0.607 \\
\hline Peripheral vasodilators & 0.549 & $(0.165-1.820)$ & 0.327 \\
\hline Selectivebeta-blockers & 1.648 & $(0.922-2.946)$ & 0.092 \\
\hline Alpha-glucosidase inhibitors & 0.924 & $(0.301-2.834)$ & 0.891 \\
\hline DPP4i & 0.546 & $(0.221-1.351)$ & 0.191 \\
\hline Insulin & 1.848 & $(0.788-4.334)$ & 0.158 \\
\hline GLP1 agonists & 1.761 & $(0.621-4.991)$ & 0.287 \\
\hline Metformin & 1.038 & $(0.483-2.232)$ & 0.924 \\
\hline Sulfonylureas & 0.824 & $(0.339-2.000)$ & 0.668 \\
\hline Thiazolidinediones & 0.642 & $(0.082-5.050)$ & 0.674 \\
\hline Bile acidsequestrants & 1.412 & $(0.275-7.243)$ & 0.679 \\
\hline Fibrates & 2.297 & $(0.458-11.529)$ & 0.312 \\
\hline Statin (high intensity) & 0.884 & $(0.315-2.481)$ & 0.814 \\
\hline Statin (low intensity) & 0.707 & $(0.089-5.598)$ & 0.742 \\
\hline Statin (moderate intensity) & 1.502 & $(0.445-5.065)$ & 0.512 \\
\hline
\end{tabular}

Note: Control variables are hypertension, diabetes mellitus, dyslipidemia, age, gender, BMI, and ASA.

Table 5: Multivariate logistic regression analysis using control variables showing the relationship between medications used to treat metabolic syndrome and surgical complications.

\section{Discussion}

In this study, we determined the impact of therapeutic agents used for the treatment of metabolic syndrome on surgical complications. We found that diuretics, NDCCB, DCCB, selective beta blockers, insulin, and GLP1 agonists showed a correlation with surgical complications in patients with ASA score $\geq 3$. Regarding the definition of metabolic syndrome, the WHO criteria for the diagnosis of metabolic syndrome are complex, because it takes into account microalbuminuria, plasma insulin level, and BMI and WC. Central obesity is a key criterion of the global consensus definition for metabolic syndrome. As our study lacked data on WC, future studies including WC criterion are warranted.

Our results demonstrated that patients with metabolic syndrome who were administered diuretics, NDCCB, DCCB, selective beta-blockers, insulin, and GLP1 agonists had more surgical complications. However, the results were equivocal, with a relatively small odds ratio when the correlation of surgical complications with each of these medications was examined. As we already know, the larger the odds ratio, the higher the statistical significance in case-control studies. To avoid bias, we also performed multivariate logistic regression using control variables, namely, hypertension, diabetes mellitus, dyslipidemia, age, gender, BMI, and ASA. The positive correlation between diuretics and surgical complications might be because of chronic potassium loss, especially in hypotension cases during general anesthesia $[13,14]$. It also emphasized the importance of perioperative fluid therapy. Switching to $\mathrm{K}+$-sparing diuretics or supplementation of potassium prior to operation may be beneficial in such cases. Alteration of GLP-1 levels might play important roles in loss of glucose homeostasis in injured patients [15]. This finding was consistent with our result, wherein a positive correlation was observed between GLP1 agonist user and surgical complications. We hypothesized that the above agents didn't result in surgical complications but they could affect the seriousness. A large series study on major surgical site infections (SSIs) in aesthetic surgery reported that the significant risk factors for postoperative SSIs included age, female gender, obesity, smoking, preexisting diabetes mellitus, trunk, and extremity procedures [16]. Breast SSIs are also major surgical complications. A systematic review identified the significant risk factors as follows: increased age, hypertension, higher BMI, diabetes mellitus, ASA3 or ASA4, previous breast biopsy or operation, pre-operative chemoradiation, conservation therapy versus other surgical approaches, hematoma, seroma, more intraoperative bleeding, post-operative drain, longer drainage time, and second drainage tube placed [17]. Our 
results were in agreement with the above findings of the systematic review.

In 2016, ICD 9 classification, which was still used in our hospital, was revised to ICD-10; however, this did not affect our assessment. Perioperative statin therapy for metabolic syndrome has gained popularity in recent years $[18,19]$. Since patients with cardiovascular operations showed a higher risk for developing surgical complications, the counteraction of statins on the detrimental effect help in combating surgical stress. In the near future, the surgical complications of metabolic syndrome, especially vascular injury, might be prevented by the available anti-diabetic medicines [20]. The urological complications (ICD9 997.5) were included in our study without exception. Urinary tract complications were no different from other systems in spite of their vast endoscopic nature. There are certain limitations to the present study. First, in subjects receiving multiple drugs or compound medicine, assessment was only performed separately for each drug. Second, this is a retrospective study with a non-experimental design and therefore, a prospective research is warranted.

\section{Conclusion}

In patients with severe diseases ( $\geq$ ASA3), the agents showing a correlation with surgical complications were diuretics, NDCCB, DCCB, selective beta blocker, insulin, and GLP1 agonists. Among them, the most prominent impact was for diuretics.

\section{Acknowledgments}

The authors thank the staff at the information technology department for their assistance.

\section{References}

1. Tzimas P, Petrou A, Laou E, Milionis H, Mikhailidis DP, et al. (2015) Impact of metabolic syndrome in surgical patients: should we bother? Br J Anaesth 115(2): 194-202.

2. Manning MW, Cooter M, Mathew J, Alexander J, Peterson E, et al. (2017) Angiotensin Receptor Blockade Improves Cardiac Surgical Outcomes in Patients With Metabolic Syndrome. The Annals of thoracic surgery 104(1): 98-105.

3. Edelstein AI, Lovecchio F, Delagrammaticas DE, Fitz DW, Hardt KD, et al. (2017) The Impact of Metabolic Syndrome on 30-Day Complications Following Total Joint Arthroplasty. J Arthroplasty 32(2): 362-366.
4. Ozkan S, Ozdemir F, Ugur O, Demirtunc R, Balci AY, et al. (2016) The effects of the metabolic syndrome on coronary artery bypass grafting surgery. Cardiovascular journal of Africa 28(1): 48-53.

5. Edelstein AI, Suleiman LI, Alvarez AP, Sacotte RM, Qin $\mathrm{CD}$, et al. (2016) The Interaction of Obesity and Metabolic Syndrome in Determining Risk of Complication Following Total Joint Arthroplasty. J Arthroplasty 31(9): 192-196.

6. Sener NC, Zengin K, Ozturk U, Bas O, Ercil H, et al. (2015) The impact of metabolic syndrome on the outcomes of transurethral resection of the prostate. J Endourol 29(3): 340-343.

7. Gage MJ, Schwarzkopf R, Abrouk M, Slover JD (2014) Impact of metabolic syndrome on perioperative complication rates after total joint arthroplasty surgery. J Arthroplasty 29(9): 1842-1845.

8. Hwang LC, Bai CH, Chen CJ (2006) Prevalence of obesity and metabolic syndrome in Taiwan. J Formos Med Assoc 105(8): 626-635.

9. Ramsay JE, Greer I, Sattar N (2006) Obesity and reproduction. BMJ 333(7579): 1159-1162.

10. Heath Promotion Administration mohaw Metabolic syndrome 2007.

11. Parikh RM, Mohan V (2012) Changing definitions of metabolic syndrome. Indian J Endocrinol Metab 16(1): 7-12.

12. Alberti KG, Zimmet PZ (1998) Definition, diagnosis and classification of diabetes mellitus and its complications. Part 1: diagnosis and classification of diabetes mellitus provisional report of a WHO consultation. Diabet Med 15(7): 539-553.

13. Bandeali SJ, Kayani WT, Lee VV, Elayda M, Alam M, et al. (2013) Association between preoperative diuretic use and in-hospital outcomes after cardiac surgery. Cardiovasc Ther 31(5): 291-297.

14. Foëx P, Sear JW (2004) The surgical hypertensive patient. Continuing Education in Anaesthesia, Critical Care \& Pain 4(5): 139-143.

15. Smith RS, Fry WR, Philp FH, Philp AS, Berry SD, et al. (2012) Mild hyperglycemia, but not glucagon-like peptide 1 predicts poor outcome after injury. Am J of Surg 204(6): 915-920.

16. Kaoutzanis C, Gupta V, Winocour J, Shack B, Grotting JC, et al. (2016) Incidence and Risk Factors for Major 


\section{Open Access Journal of Urology \& Nephrology}

Surgical Site Infections in Aesthetic Surgery: Analysis of 129,007 Patients. Aesthet Surg J 37(1): 89-99

17. Xue DQ, Qian C, Yang L, Wang XF (2012) Risk factors for surgical site infections after breast surgery: A systematic review and meta-analysis. Eur J Surg Oncol 38(5): 375-381.

18. Putzu A, Capelli B, Belletti A, Cassina T, Ferrari E, et al. (2016) Perioperative statin therapy in cardiac surgery: a meta-analysis of randomized controlled trials. Crit Care 20(1): 395.

19. Chan WWN, Wong GTC, Irwin MG (2013) Perioperative statin therapy. Expert Opinion on Pharmacotherapy 14(7): 831-842.

20. Dominguez LJ, Sowers JR (2005) Metabolic syndrome therapy: prevention of vascular injury by antidiabetic agents. Curr Hypertens Rep 7(2): 110-116. 